\title{
Cambiar la burocracia y el control por la formación y la confianza*
}

\begin{abstract}
Milton Calderón-Vélez ${ }^{1}$
Centre de recherches interdisciplinaires, Démocratie, Institutions, Subjectivité (CriDIS) - Universidad Católica de Lovaina, Bélgica Facultad Latinoamericana de Ciencias Sociales, FLACSO Ecuador milton.calderon@uclouvain.be
\end{abstract}

Artículo recibido en noviembre y aprobado en diciembre 2020

\section{Resumen}

En el siguiente ensayo se explora la tensión, visible en el sistema educativo ecuatoriano, entre un tipo de gestión basado en la burocracia y el control y otro basado en la formación y la confianza, este último cada vez más demandado por las y los educadores. El ensayo busca comprender las lógicas de trabajo imperantes en el marco de la pandemia de la COVID-19, toda vez que la misma ha visibilizado una serie de problemáticas educativas persistentes a lo largo de los años pero que aparecen con más fuerza en momentos de incertidumbre. La primera parte explica el contexto desde el cual se hace la lectura de las respuestas educativas en tiempos de pandemia, y la segunda presenta, a breves rasgos, los dos sistemas de gestión que dan cuenta de la tensión entre las miradas estandarizadas, promovidas por el Estado, y las demandas de confianza de los educadores. Al final, se enlistan algunas alternativas para hacer cada vez más presente este segundo tipo de gestión, y caminar hacia una educación que se libere de las lógicas que la han aprisionado a lo largo de los años.

Palabras clave: estandarización, control, confianza, formación, Covid-19

1 Doctorando en Ciencias Políticas y Sociales en la Universidad Católica de Lovaina. Magister en Sociología por la Facultad Latinoamericana de Ciencias Sociales FLACSO, Ecuador. Diplomado Superior en Investigación Social por el Consejo Latinoamericano de Ciencias Sociales CLACSO, Argentina. Licenciado en Ciencias de la Educación por la Pontificia Universidad Católica del Ecuador PUCE. Estudios superiores de filosofía y cine. Desarrollo de investigaciones y publicaciones académicas, entre otras. Trayectoria como docente, coordinador de proyectos, formador de juventudes y educadores y consultor.

* Una parte de este ensayo fue publicado en forma de pequeños textos en la página web www.sociotramas.org. 


\section{Switching from bureaucracy and control to training and trust (1)}

\section{Abstract}

The following essay explores the tension, visible in the Ecuadorian educational system, between a type of management based on bureaucracy and control and another based on training and trust, the latter increasingly demanded by educators. The essay aims to understand the prevailing logics of work in the framework of the COVID-19 pandemic, since it has made visible a series of educational problems that have persisted over the years but that appear more strongly in times of uncertainty. The first part explains the context from which the educational responses are read in times of pandemic, and the second part presents, in brief, the two management systems that account for the tension between the standardized views, promoted by the State, and the demands of trust from educators. In the end, some alternatives are listed to make this second type of management more and more present, and to move towards an education that frees itself from the logics that have imprisoned it over the years.

Keywords: standardization, control, trust, training, Covid-19.

\section{Introducción}

El 15 de marzo de 2020, al iniciarse la implementación de medidas de control sanitario por la pandemia de la Covid-19, el Ministerio de educación del Ecuador dispuso la continuación de las actividades escolares en la modalidad de teletrabajo. ${ }^{2}$ Posteriormente, emitió los "Lineamientos e instructivo para registro y seguimiento de actividades de los funcionarios bajo la modalidad de teletrabajo", en el que explicita los pasos que deben seguir las y los educadores para registrar sus jornadas en un sistema habilitado para el efecto, so pena de sanciones administrativas.

En el mencionado instructivo, se lee:

El funcionario público que se encuentre actualmente desempeñando funciones en las instituciones educativas del

2 Acuerdo Ministerial Nro. MINEDUC-MINEDUC-2020-00014-A 
país bajo cualquier modalidad laboral, deberán realizar de manera obligatoria el registro de actividades bajo el sistema de teletrabajo, a través de un formulario virtual que permitirá al docente registrar sus actividades realizadas durante la emergencia sanitaria por el COVID 19; el mismo deberá ser llenado una vez terminada la jornada semanal (Lineamientos e instructivo para registro y seguimiento de actividades de los funcionarios bajo la modalidad de teletrabajo, 2020).

El instructivo continúa mencionando los días de la semana en que se habilitarán los formularios de acuerdo con las zonas, delegando las responsabilidades inmediatas a los funcionarios de menor rango, y explicando que, en el caso de no contar con un buen servicio de Internet, deberá llenarse un PDF que será posteriormente enviado a la autoridad educativa correspondiente. Finalmente, se dedican varias páginas a indicar cómo ingresar a la plataforma, cómo llenar los datos, una encuesta, y el registro documentado de las actividades desarrolladas a lo largo de la semana.

Este registro "obligatorio", que debe contar con los respaldos digitales de las actividades desarrolladas-los cuales podrían ser solicitados posteriormente por las autoridades a cargo-, y ser motivo de sanción en el caso de que los docentes hayan ingresado "información y datos de manera fraudulenta" da cuenta de una de las mayores preocupaciones del Ministerio de educación, la cual es controlar el trabajo de quienes ejercen la docencia a nivel nacional.

Es una muestra, también, de la desconfianza con la cual las autoridades educativas conciben la labor de los educadores -a quienes no se les puede dejar a la deriva-, multiplicando, de esta manera, los instrumentos burocráticos cuya única consecuencia es la de alejar a los profesores de la que debería ser su preocupación primordial: la enseñanza. $Y$, aunque parezca un caso aislado y desde cierto punto de vista, incomprensible, lo cierto es que la docencia en el Ecuador es un auténtico ejercicio de administración a pequeña escala, y los educadores llevan años luchando porque la autoridad educativa nacional reduzca estas cargas laborales en función de una mayor dedicación pedagógica. ${ }^{3}$

3 De hecho, es importante notar que a este instructivo le preceden varios acuerdos para reducir las cargas administrativas, todos ellos sin resultados aparentes, como por ejemplo el Acuerdo Nro. MINEDUC-MINEDUC-2019-00011-A del 20 de febrero de 2019, que emite "lineamientos para la reducción de las actividades administrativas de los docentes en el Sistema Educativo Nacional". 


\section{El contexto de la pandemia y la educación en Ecuador}

Para poder comprender de mejor manera lo expuesto, cabe considerar algunos aspectos generales de lo que ha representado la pandemia de la Covid-19 para la educación, en el marco de una cada vez mayor estandarización de los procesos educativos, en consonancia con las dinámicas globales que ahondan más y más este tipo de miradas.

El Sars-CoV-2, simplemente llamado "coronavirus", inició como una crisis de tipo sanitario, que poco a poco fue volviéndose social, económica y política, penetrando espacios en los que inicialmente se asumía no tenía mayor incidencia. Así, vemos cómo en muchos países del mundo comenzaron a desatarse olas de protestas por las medidas adoptadas (INFOBAE, 2020; RTVE, 2020), algunas a favor otras en contra, o bien, a destacar las latentes miradas xenofóbicas y racistas al vincular al virus con un segmento específico de la población mundial (Tavernise \& Oppel, 2020). Esto, como era de esperarse, excedió las preocupaciones meramente sanitarias, y llevó las discusiones sobre el tema al terreno de lo social.

Posteriormente, esta crisis llegaría al campo económico, pues la implementación de las medidas de confinamiento y el consecuente freno de las industrias y comercios provocaron una crisis sin precedentes en la historia global (Banco Mundial, 2020), misma que aún se encuentra en curso. Por supuesto, un país como Ecuador, no podría estar lejano de estas diversas problemáticas, aunque para el caso, muchas de ellas no fueron inauguradas por la pandemia, sino más bien visibilizadas por la misma. De ahí que, durante la etapa de mayor complejidad de la situación, en el país comenzaron a destaparse varios casos de corrupción ligados, precisamente al ámbito de la salud, en el que destacan el reparto de los hospitales por cargos públicos, el desvío de implementos médicos y la existencia de fraudulentos carnés de discapacidad (Ecuavisa, 2020), además de la brillante idea del entonces Ministro de economía de pagar la cantidad de 325 millones de dólares a los tenedores de deuda externa dejando impagos a los servidores públicos, y entre ellos al personal de salud, y a los docentes. 
De esta manera, la crisis sanitaria de la Covid-19, ahora social, política y económica se convirtió en el agente por el cual se tornaron visibles algunas problemáticas que por las circunstancias de aparente "normalidad", no habían sido tratadas en el ámbito de lo político, -aunque muchas de las veces expuestas en el ámbito de lo académico-. Es así como llegamos al terreno de la educación, en el que una crisis compleja se puso en evidencia al poner a prueba las prácticas educativas en un contexto de incertidumbre y creciente complejidad.

De la noche a la mañana vimos cómo las autoridades educativas nacionales, comenzaron a experimentar algunas respuestas posibles a la crisis, tratando inicialmente de llevarlo todo al ámbito de lo virtual, cuestión que pronto se verá limitada por la constatación de que únicamente el $45 \%$ de la población ecuatoriana cuenta con acceso a Internet (Dávalos, 2020a), y que además este casi no llega a los sectores rurales y las provincias con menos habitantes.
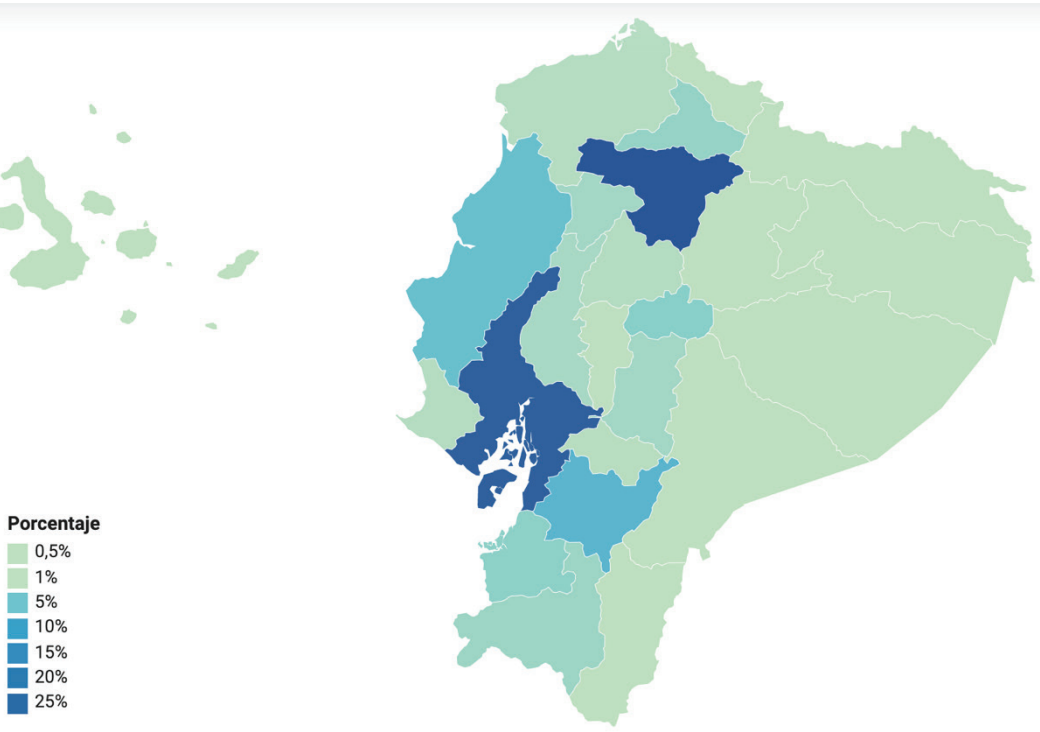

Figura 1. Mapa con el porcentaje de cuentas de Internet fijo por provincia hasta junio de 2020. Fuente principal ARCOTEL, en Dávalos (2020b)

A esto podría sumarse la explosión de capacitaciones 
emergentes ligadas al uso de tecnologías de la información, la lenta respuesta ministerial para adaptar los procesos de enseñanza a otro tipo de mecanismos de difusión (como por ejemplo a la radio y la TV), o la aún limitada reflexión en torno a la necesidad de adaptar el currículo a nuevas circunstancias o a abrir el espectro educativo a otras modalidades (como aquellas de educación en casa o de modalidad virtual). ${ }^{4}$

Es importante mencionar que, como en otros países del mundo, la preocupación por las tecnologías de la información y por su inclusión en los procesos educativos no es nueva. Si se hubiese tomado con mayor seriedad la Cuarta revolución industrial caracterizada por la cada vez mayor digitalización de la vida cotidiana, y la híper conexión con el mundo (Schwab, 2016), muy probablemente no habrían tenido que darse respuestas improvisadas a una situación de emergencia. Sin embargo, la pandemia de la Covid-19, puso a todo el mundo a correr, y en la práctica entorpeció los procesos pedagógicos, que incluso haciendo uso de aplicaciones como WhatsApp buscan hoy ser sostenidos por los educadores. ${ }^{5}$ Las brechas tecnológicas, pero también sociales, que obligaban a los habitantes a salir de sus hogares en tiempos de confinamiento, seguían irresolutas con los mecanismos implementados para salvar en algo la educación en tiempos de Covid-19.

En este contexto, ¿es posible pensar que las medidas para mantener las dinámicas de control burocrático, como aquellas presentadas en la introducción, son acertadas? En definitiva, lo que estas hacen es poner un nuevo problema sobre los hombros de las y los educadores, muchos de los cuales aún están lidiando por sostener su propia salud mental o la situación económica que les provocaron las medidas de reducción salarial, introducidas para "salvar la situación" por el gobierno de turno. En todo caso, la velocidad con la que aparecieron las normas dedicadas a controlar el trabajo de los docentes en casa,

4 Si bien se abrieron caminos para llevar los procesos educativos con modalidades diferentes, y se hizo una adaptación curricular denominada "currículo priorizado", estas siguen siendo limitadas pues no logran responder a los diferentes contextos. Los docentes y educadores en general, siguen manifestando inconformidad sobre estos procesos que no logran empatar con las demandas, probablemente porque se los desarrolla sin contar con la voz de los actores.

5 Docentes de sectores rurales me comentan que la plataforma que más utilizan para tratar de mantener sus clases es la aplicación de mensajería WhatsApp. 
versus la lentitud para comprender las otras problemáticas y llevar adelante soluciones plausibles, se convierte en una tensión que vale la pena considerar con seriedad.

\section{Dos lógicas en tensión}

Se ha iniciado hablando de dos distintos modelos de gestión, uno de los cuales está signado por las lógicas de la burocratización y la necesidad de control que este genera, y otro motivado por la formación y la confianza -del cual aún pareciera que estamos lejos-. Estos dan forma a una evidente tensión que se visibiliza en el espacio de la gestión de los espacios educativos, y que jala hacia uno u otro lado, transformando las prácticas de los educadores, y definiendo las mismas al subsumirlas o no a la necesidad del cumplimiento.

El primero de estos modelos, hemos colocado en el lado de la política educativa, aunque vale aclarar, llegados a este punto, que no se trata de un análisis polarizado de la realidad, que divide las dinámicas entre buenas y malas, objetivando a los actores y otorgándoles características que no poseen, sino de una división estrictamente metodológica que trata de dar cuenta de los elementos empíricos que se pueden observar en el terreno educativo. ${ }^{6}$ Por lo tanto, es notable considerar que aún con las lógicas de burocracia y control que se definen por medio de políticas educativas estandarizadas, estas suelen aterrizar en la práctica pedagógica de muchas formas, dependiendo, más bien, de cuánto una persona ha asumido o no dichas lógicas en su trabajo cotidiano (Ceulemans, 2017). Sin embargo, se considera, también, que el hecho de que muchas de estas normas vengan acompañadas de sus consecuentes sanciones resta capacidad a los educadores para actuar libremente y gestionar lo imprevisto (Mejía, 2001).

Dicho esto, cabe manifestar que el primero de estos tipos de gestión, asume como unidad clave al estándar, en consonancia con

6 Otra aclaración que cabe hacer aquí, es que la estandarización no es un asunto únicamente del Estado, sino que aterriza de formas diversas en las organizaciones educativas, que van generando también dispositivos de control, en el marco de la misión que han asumido. Podría hablarse de distintos niveles de estandarización que afectan, finalmente, el trabajo cotidiano de las y los educadores. 
las dinámicas globales que van orientándose en el mismo sentido. El estándar es un concepto estadístico en el que se representan variables a medir y frecuencias, convirtiendo un conjunto de valores en un rango referencial, que se denominar "curva normal" o "campana de Gauss". Esto resulta relevante en la medida en que la idea de estandarización pasa por la noción de "normalidad" o de medida estándar, de manera que aquello que se ubique por debajo de la misma, o muy por encima de esta, será considerado "anormal", en el sentido de que rompe la "norma ideal de conducta" sobre la cual se premia a unos y se castiga a otros (Foucault, 1981, 2009).

Ahora, si bien es cierto que la estandarización es útil en muchos de los aspectos de la vida de un Estado, sobre todo porque facilita la eficiencia y la eficacia de las instituciones, es necesario indicar que en el ámbito de la educación, la misma tiene consecuencias importantes, dado que "la lógica de los números se filtra a variables cualitativas, forzando su cuantificación y derivando en un paradigma obediente a números, indicadores y estándares" (Molina, 2017, p. 71), cuestión que no solo toca el ámbito de la de gestión, sino que aterriza en los espacios en los que se desarrolla la práctica más pedagógica y didáctica.

La política educativa en el Ecuador, no ha permanecido lejana de esta mirada que propende a comprender como "normales" algunos aspectos de la realidad, aunque dicha normalidad sea concebida al interior de espacios muchas de las veces lejanos de aquellos en los que se los aplica. Es en este sentido que los estándares son construcciones, además concebidas al margen de los contextos, valga decir, en este caso, al margen de los centros escolares, y en la mayoría de las veces sin las voces de los estudiantes, docentes, autoridades, y demás actores que existen en el espacio educativo.

En consecuencia, cuando se habla de estandarización se está haciendo referencia a una realidad compleja que concibe ciertos aspectos de la realidad dentro de un rango de aparente normalidad, construida de manera externa, cuyos efectos prácticos son la "introducción" o bien la "exclusión" de un determinado sistema. Estas diferentes técnicas o tácticas que van estableciendo rangos "normales", se tornan además una manera de gestionar los diferentes procesos que existen, sobre todo 
en materia educativa. Dicho de otro modo, la estandarización, en su afán de normalizar, propenderá a medir dicha normalidad, estableciendo los mecanismos para ello.

Este es el origen de la burocracia en la escuela, de modo que los intentos por reducirla, y las demandas de los educadores por alejarse de ella, resultan en gran medida inútiles, al no poder romper con la lógica que mueve estos esfuerzos y que se ubica en la estandarización. Por ende, estamos aún lejos de la aplicación del currículo que "abre puertas" a una mayor contextualización, y que declara que: "Las instituciones educativas disponen de autonomía pedagógica y organizativa para el desarrollo y concreción del currículo, la adaptación a las necesidades de los estudiantes y a las características específicas de su contexto social y cultural"(MINEDUC, 2016, p. 15).

Así , la estandarización (en el currículo) genera una suerte de modelización del proceso educativo, en tanto responde a criterios externos, y no necesariamente pensados por el docente, lo cual favorece la imagen del mismo como un técnico, y no como un científico de la educación (Giroux, 1995, 1997), imagen que se irá trasladando también a los estudiantes y sus familias, quienes, por esta razón, asumirán que pueden dar orientaciones a los maestros en las tareas que aquellos consideran "no hacen bien".

Esto le quita valor a la función docente, un trabajo en el que la vocación juega un rol definitivo, y en que la creatividad es un recurso cotidiano. El círculo vicioso de la mirada estandarizada, la visión del docente como técnico, y el interés por controlar el currículo, no tendrá otra consecuencia que no sea la existencia de nuevas formas de burocratización, nuevas «cargas», como la que se han presentado al iniciar este ensayo, pues la estandarización siempre se encargará de multiplicar los mecanismos para controlar el cumplimiento de la normalidad.

Pese a ello, es notable que los sistemas educativos que mejor han funcionado son los que se han encargado de controlar menos y formar más. Las y los docentes necesitan de procesos de formación sostenidos en el tiempo, y pensados en el marco de un proyecto 
educativo a mediano y largo plazo. ${ }^{7}$ El tratar de tapar cada problema con capacitaciones que hacen pequeños remedos, es una forma de atacar los síntomas sin llegar nunca a entender la enfermedad.

Los actuales proyectos que tienen como fin la revalorización de los docentes, olvidan que una de las cuestiones clave para que esto sea posible es la de mantener la confianza en que los profesores son capaces de hacer un trabajo de calidad, aún sin la necesidad de que otros funcionarios les "hagan sombra" ${ }^{1}$, pero para que esto sea posible debe garantizarse que la formación de los educadores responda a las nuevas circunstancias y les permita tomar sus propias decisiones. Esto es probablemente lo que hace tan atractiva a la propuesta finlandesa de educación, pues detrás de las ideas muchas veces compartidas de no tener inspecciones, exámenes, o evaluaciones de profesores, se encuentra todo un programa orientado a que los mejores profesionales sean docentes, y a que por supuesto, tengan sueldos justos. Nuevamente, el ideal no podría hacerse realidad, sin que se vuelque la mirada a los aspectos más esenciales de la vida de las personas, pues solo podrá ser posible la revalorización, en la medida en que el valor con el que se mira a los educadores se visibilice en las políticas que se crean para sostenerlos.

Un sistema orientado por la formación y la confianza tendría que ser capaz de construir espacios pedagógicos en los que prime la necesidad de compartir aquello que se va construyendo en el camino -dado que no hay espacio de mayor innovación que el educativo-. A esto han apuntado iniciativas que vinculan la docencia a las dinámicas de investigación-acción (Fals Borda, 1993; Ortiz \& Borjas, 2008) y que han surgido de las bases de la pedagógica critica con el fin de convertir a los educadores en "intelectuales transformativos" (Giroux, 1997). Por supuesto, un sistema que rompa con la idea de un educador cuya tarea es la de cumplir paso a paso con una agenda no pensada por él mismo,

7 Se hace aquí una pequeña distinción entre formación y capacitación, asumiendo la primera como un proceso sostenido, y la segunda como una solución emergente y de corta duración.

8 En medio de un proceso de investigación, una psicóloga de un importante centro educativo en la ciudad de Quito, utilizó la expresión "hacer sombra", para definir las estrategias implementadas por su departamento para vigilar a los estudiantes en todo momento. Es una excelente metáfora para definir las intenciones que subyacen a las prácticas de control del Ministerio de educación. 
favorecerá la reflexión sobre la propia acción y alejará a los docentes de esta mirada de técnicos y burócratas al servicio de instituciones, más que de personas que buscan aprender.

La formación de los educadores habría de ser la base por la cual atraviesen todas las políticas en materia educativa. Quizá el tiempo de pandemia pudo ser una valiosa oportunidad para "detenerlo todo", y formar a los educadores, no ya en cuestiones que permitan superar las circunstancias coyunturales, es decir, ligadas al uso de tecnologías, sino en torno a principios epistemológicos, filosóficos, pedagógicos y didácticos que les orienten a reflexionar sus propias prácticas y a reinventarlas a partir de dichas reflexiones.

Hoy, probablemente estamos ya tarde, para aprovechar de mejor manera el desorden provocado por la crisis sanitaria, aunque siempre se puede comenzar a estructurar un plan a largo plazo que revolucione el ejercicio de la docencia en el Ecuador. En todo caso, caminar de esta lógica de burocracia y control a una de formación y confianza, parece ser un camino largo de recorrer, pero, sin lugar a duda, uno de los más necesarios.

\section{Algunas pistas para hacer camino}

Como se ha expresado a lo largo de este ensayo, las y los educadores se encuentran en medio de un sistema cuya base es la burocracia y el control. Lamentablemente, salir de este resulta complejo pese a las constantes demandas de los educadores por hacerlo, y requerirá de tiempo para garantizar su adecuada formación y profesionalización, además del evidente cambio estructural que implica movilizar nuevas alternativas desde instituciones como el Ministerio de educación.

Sin embargo, se ha querido subrayar en el texto, que este es un camino adecuado para hacerlo, más aún en el contexto actual, en que la pandemia de la Covid-19 ha puesto en evidencia la profunda crisis de nuestros sistemas educativos, las brechas tecnológicas que aún debemos superar, y las tensiones que existen entre los modelos de gestión propuestos y los ideales de los educadores. 
Mientras tanto, sirvan algunas pistas para hacer camino desde los espacios de la docencia y la gestión.

\section{Desde la docencia}

\section{¿Qué hacer?}

1. Aprender, sin miedo, de las circunstancias. Sea con plataformas de videoconferencia, sea con aplicaciones de mensajería, estamos en un momento en el que nos vemos obligados a interactuar con los otros mediados por tecnologías. Ello convierte a la coyuntura en un excelente momento para aprender y dejar de tenerle miedo a las opciones que el nuevo siglo nos ofrece para educar.

2. Explorar herramientas y recursos en línea. Ligado al hecho de que la crisis tiene una importante dimensión de aprendizaje, está el hecho de que la información abunda. Por lo tanto, es un buen momento para explorar herramientas que pueden sernos útiles, no solo durante la crisis actual, sino a futuro. Aquí vale la pena tomar en cuenta algunas recomendaciones como: verificar que la información no aparezca en páginas anunciadas, usar varias palabras clave en las búsquedas para hacerlas más específicas, verificar que las páginas cuenten con autor y fuentes, fijarse en las fechas de actualización de la información, $y$, finalmente, preferir páginas que hablen de educación para evitar utilizar contenido meramente comercial.

3. Evitar "llevarse el trabajo a casa" aún en casa. Aunque pueda parecer paradójico pues varias de las actividades las hacemos hoy en casa, ${ }^{9}$ es importante dividir los tiempos de modo que la cuestión laboral no sea la única tarea a la que nos dediquemos, desaprovechando así la posibilidad de compartir más con nuestros familiares, llamar a nuestros amigos, o iniciar nuevos proyectos personales.

9 Es evidente que la realidad no es la misma para todas las personas, y que es muy relativo hablar del "trabajo en casa" para todos, o en las mejores condiciones posibles. Sin embargo, se presentan estas líneas en coherencia con el caso inicial, asumiendo que los docentes están "obligados" a hacer "teletrabajo". Por supuesto el ensayo no aborda, de momento, las desigualdades presentes en el campo de la docencia, y visibles, también durante la pandemia. 


\section{¿Qué no hacer?}

1. Llevar la modalidad presencial a la virtual. Uno de los errores pedagógicos más comunes es el de hacer lo mismo que haríamos en una clase presencial, pero utilizando medios virtuales. Es importante entender que el medio virtual requiere también ciertas adaptaciones como, por ejemplo, reducir un poco los tiempos, dosificar los contenidos y explotar más las herramientas virtuales, sobre todo porque una modalidad de este tipo, siempre resulta más cansada.

2. Excederse con las tareas. Otro error es el hecho de excederse con tareas asumiendo que los estudiantes tienen más tiempo para hacerlas. Si asumimos que todos los docentes piensan lo mismo lo que tendremos son estudiantes hartos de los deberes y mucho más desmotivados para el aprendizaje. Es preferible dejar ciertas tareas mentales, que lancen a los estudiantes a la curiosidad, en lugar de tareas cuyo fin sea "tener evidencia de que cumplimos con nuestro trabajo"

3. Trasladar la responsabilidad pedagógica a padres y madres. Mal haría un educador si asume que los padres y madres de familia tienen la responsabilidad de garantizar el aprendizaje en estos tiempos en los que el hogar se ha convertido en un aula. Es importante saber que, aún en tiempos de crisis, nuestro trabajo sigue siendo el de educadores. Una de las mejores acciones para valorizar nuestra profesión es la de continuar haciendo nuestro trabajo con la misma pasión de siempre. Además, ¿por qué no aprovechar para reunirnos con los padres de familia con la finalidad de que aquellos se inmiscuyan más en la vida estudiantil de sus hijos?

\section{Desde la gestión}

\section{¿Qué hacer?}

1. Buscar mecanismos para la formación de los profesores. Si los profesores podrían aprovechar estos momentos para relacionarse mucho más con los padres, más allá de los clásicos 
momentos de entrega de reportes de calificaciones, ¿por qué no aprovechar desde la escuela para garantizar la formación de los educadores? No hace falta ir lejos ni contratar expertos, pues entre los mismos profesores podría aprovecharse los puntos fuertes de cada uno o hacer uso de espacios abiertos que se orientan a la formación. Una reducción de las actividades "de clase" en virtud de un aumento de actividades de aprendizaje de los maestros no solo sería necesario sino, además, justo.

2. "Acompañar" a toda la comunidad educativa. En el sentido más profundo que le podamos dar al término "acompañamiento", este es un momento en que menos solos debe dejarse a los docentes y a la comunidad educativa. Estos tienen familias, problemas, circunstancias con las que deben combinar sus tareas laborales, y por ende necesitan del apoyo de quienes están al frente de los centros educativos. Conversar con ellos, comprender las situaciones que atraviesan, y apoyarles en la medida de lo posible es un buen comienzo para que aquellos sientan que están "sostenidos" en momentos de crisis.

3. Ser propositivos para la acción educativa. Si bien el docente está llamado a buscar alternativas, herramientas, recursos, documentos, y otros que modifiquen su accionar pedagógico, es cierto que esta no debe ser únicamente su responsabilidad. O más bien dicho, las autoridades, desde su posición pueden aportar significativamente a esta búsqueda. Además, son quienes probablemente reciben más información que puede resultar útil y que podría compartirse con ellos.

\section{¿Qué no hacer?}

1. Aumentar la burocracia orientada al control. Como se ha expresado, muchos educadores deben hacer juego entre sus actividades de docencia y sus responsabilidades de padres y madres, y, lamentablemente, este juego tiene la desventaja de que lo primero está usualmente orientado por la lógica del cumplimiento. Muchos directores pueden estar tentados a aumentar la burocracia en estos días, bajo el pretexto de contar con "fuentes de verificación" de un trabajo que se desarrolla 
desde casa. Lo que obtenemos es un montón de docentes con una carga más, menos preocupados por lo pedagógico, y, además, un montón de estudiantes cargados de tareas.

2. Restar valor al trabajo de quienes educan. Los padres y madres de familia han de comprender que la labor de los docentes va mucho más allá del tiempo que duran estas. La planificación, y todos estos elementos que suponen para ellos una carga innecesaria de trabajo deben considerarse en su valorización. En este momento es importante que las autoridades promuevan campañas que hagan visible el fuerte trabajo de los educadores, en lugar de minimizar el mismo, incluso, muchas veces, puertas adentro.

3. Sancionar. Es importante entender que las circunstancias actuales son excepcionales, y aunque el mismo Ministerio haga de la sanción su política educativa, se debe comprender las dificultades y situaciones complejas que hoy viven muchos educadores. Lo mejor es evitar la sanción, y como se dijo antes, aumentar el acompañamiento. En nuestras manos está, sobre todo en tiempos de crisis global, responder con una buena carga de humanidad.

\section{Conclusión}

Se ha iniciado este ensayo poniendo sobre la mesa un caso particular que puede ser el centro del debate y que explica el modo en que el Ministerio de educación del Ecuador, prioriza las acciones ligadas a la burocracia y el control, disminuyendo la capacidad creativa y de respuesta pedagógica que pueden tener las y los educadores. Esto en el marco de la crisis del sistema que se torna más visible con la pandemia de la Covid-19, y que ha puesto en evidencia la necesidad de contar con proyectos educativos a mediano y largo plazo que coloquen los procesos de enseñanza y aprendizaje por encima de los intereses políticos de turno.

Posteriormente, se ha presentado algunos factores que definen esta crisis, y el modo en que estos han sido poco considerados en las respuestas a nivel educativo. Al menos en un primer momento, las 
respuestas no resultaron ser las más efectivas, y la preocupación se trasladó a la necesidad de controlar el cumplimiento del trabajo de los educadores en sus casas, en lugar de garantizar que los procesos educativos se sigan llevando a cabo y no se merme la ya disminuida calidad de nuestro sistema.

En una segunda sección se ha presentado la tensión que suponen estos dos modos de concebir la gestión en tiempos de pandemia, haciendo énfasis en las consecuencias de las lógicas estandarizadas y orientadas al control para los procesos educativos en general. Al hablar de una tensión, lo que se ha querido es mostrar cómo los docentes se ven inmersos en un espacio de trabajo cuyo fin está signado por la labor técnica de responder y consolidar fuentes de verificación para mostrar a unos funcionarios que poco o nada entienden de los contextos educativos en los que estos son producidos, motivando a que las prácticas sean cada vez menos pedagógicas, y al mismo tiempo más burocráticas.

Esta tensión se resuelve en última instancia en el plano de la acción de las y los educadores, toda vez que se trata de acciones heterogéneas, y pone en evidencia la demanda creciente por reducir las "cargas administrativas", y tener mayor confianza en el trabajo que desempeñan los educadores. Por supuesto, esto solo será posible en la medida en que, a nivel estatal y de los centros educativos se garanticen procesos fuertes de formación, y no únicamente capacitaciones espontáneas.

Para concluir se han presentado algunas pistas para hacer camino hacia este segundo modo de gestión, considerando para ello tanto el espacio de la docencia como el de la gestión, y motivando a que las pequeñas acciones se encaminen a fortalecer un proceso que, sin lugar a dudas, no podrá extenderse sin la voluntad política. Sea entonces, una oportunidad para colocar estos temas en la agenda educativa de los próximos años, y comenzar a construir un sistema cuyo eje sea la confianza, y que garantice la formación de educadores para un nuevo y complejo siglo. 


\section{Bibliografía}

Banco Mundial. (2020, junio 8). La COVID-19 (coronavirus) hunde a la economía mundial en la peor recesión desde la Segunda Guerra Mundial. Comunicado de prensa. https://www.bancomundial.org/ es/news/press-release/2020/06/08/covid-19-to-plunge-globaleconomy-into-worst-recession-since-world-war-ii

Ceulemans, C. (2017). How to move beyond the trust - control dilemma? What insight into the work of educational standards might have to offer. Studia paedadogica, 22(2).

Dávalos, N. (2020a, agosto 29). Solo el 45.5\% de hogares en ecuador tiene acceso a internet, según el INEC. PRIMICIAS Tecnología. https://www.primicias.ec/noticias/tecnologia/ecuador-hogaresacceso-internet-inec/

Dávalos, N. (2020b, septiembre 12). Ecuador tiene 11,18 millones de conexiones a internet fijo y móvil. PRIMICIAS Tecnología. https://www.primicias.ec/noticias/tecnologia/ecuador-millonesconexiones-internet-fijo-movil/

Ecuavisa. (2020, mayo 28). Corrupción: la otra pandemia que golpea a Ecuador / Ecuavisa. Noticias. https://www.ecuavisa.com/articulo/ noticias/nacional/605851-corrupcion-otra-pandemia-que-golpeaecuador

Fals Borda, O. (1993). La investigación participativa y la intervención social. Documentación Social. Revista de estudios Sociales y de sociología aplicada, 92, 9-21.

Foucault, M. (1981). Las Mallas del Poder. Paidós Ibérica.

Foucault, M. (2009). Vigilar y castigar. Nacimiento de la prisión. En Vigilar y castigar. Nacimiento de la prisión. Siglo XXI Editores.

Giroux, H. (1995). Teoría y resistencia en educación. Una pedagogía para la oposición. En Docencia (Vol. 15). Siglo XXI Editores.

Giroux, H. (1997). Los profesores como intelectuales transformativos. Docencia, 15, 60-66. 
INFOBAE. (2020, marzo 25). Bolsonaro critica cuarentena por coronavirus y pide "vuelta a la normalidad" de Brasil - Infobae. AGENCIAS. https://www.infobae.com/america/agencias/2020/03/25/ bolsonaro-critica-cuarentena-por-coronavirus-y-pide-vuelta-a-lanormalidad-de-brasil/

Mejía, M. (2001). Educaciones y pedagogías críticas desde el sur (Cartografías de la Educación Popular). CEAAL, Consejo de educación de adultos de América Latina.

Lineamientos e instructivo para registro y seguimiento de actividades de los funcionarios bajo la modalidad de teletrabajo, (2020).

Ortiz, M., \& Borjas, B. (2008). La Investigación Acción Participativa: aporte de Fals Borda a la educación popular. Espacio abierto. Asociación Venezolana de Sociología, 17, 615-627.

RTVE. (2020, mayo 9). Coronavirus I Miles de personas protestan en Alemania contra las medidas restrictivas por el coronavirus. Coronavirus. https://www.rtve.es/noticias/20200509/milespersonas-protestan-alemania-contra-medidas-restrictivascoronavirus/2013717.shtml

Schwab, K. (2016). The Fourth Industrial Revolution: what it means and how to respond. World Economic Forum, 01. https://www. weforum.org/agenda/2016/01/the-fourth-industrial-revolutionwhat-it-means-and-how-to-respond/

Tavernise, S., \& Oppel, R. (2020, marzo 23). Les escupen, les gritan, los atacan: los chinoestadounidenses temen por su seguridad The New York Times. Estados Unidos. https://www.nytimes.com/ es/2020/03/23/espanol/mundo/coronavirus-racismo.html 